Pacific Journal of Mathematics

RICCI CURVATURE AND VOLUME GROWTH

GE AND GERARD WATCH 


\title{
RICCI CURVATURE AND VOLUME GROWTH
}

\author{
M. Strake and G. Walschap
}

We give an example of a complete manifold $M^{m}$ of nonnegative Ricci curvature for which the volume of distance tubes around a totally geodesic submanifold $L^{l}$ divided by the corresponding volume in $L \times \mathbf{R}^{m-l}$ goes to infinity. Recall that in the case of nonnegative sectional curvature, this quotient is nonincreasing and bounded by 1 .

1. Introduction. One of the fundamental tools in the study of Ricci curvature is the Bishop-Gromov volume inequality, which states that in a complete manifold $M^{m}$ of Ricci curvature $\geq(m-1) \kappa$, the map

$$
r \mapsto \frac{\operatorname{vol} B_{r}(p)}{\operatorname{vol}\left(D_{r}, \hat{g}_{\kappa}\right)}
$$

is monotonically nonincreasing. Here, $B_{r}(p)$ is the ball of radius $r$ around $p \in M$, and $\left(D_{r}, \hat{g}_{\kappa}\right)$ is a ball of same radius in the simply connected space of constant sectional curvature $\kappa$. Under somewhat different assumptions, this inequality still holds when $p$ is replaced by a compact, totally geodesic submanifold $L^{l}$ of $M$ : The comparison space now becomes $\left(L \times D_{r}, g_{\kappa}\right)$, where for $x=\left(x_{0}, x_{1}\right)$ in the tangent space of $L \times D_{r}$ at $(p, u), g_{\kappa}(x, x)=c_{\kappa}^{2}(|u|) \check{g}\left(x_{0}, x_{0}\right)+$ $\hat{g}_{\kappa}\left(x_{1}, x_{1}\right)$. (Here $\breve{g}$ is the metric on $L$ induced by the imbedding $L \hookrightarrow M$, and $c_{\kappa}$ is the solution of the equation $c_{\kappa}^{\prime \prime}+\kappa c_{\kappa}=0$, with $c_{\kappa}(0)=1, c_{\kappa}^{\prime}(0)=0$.) The volume inequality now reads (cf. [4], [3], [6]):

(*) If the radial sectional curvatures of $M$ are $\geq \kappa$, then

$$
q_{L}(r) \stackrel{\text { def }}{=} \frac{\operatorname{vol} B_{r}(L)}{\operatorname{vol}\left(L \times D_{r}, g_{\kappa}\right)}
$$

is a nonincreasing function of $r$, with $q_{L}(0)=1$. (A 2-plane $\sigma \subset M_{q}$ is said to be radial if it contains the tangent vector of some minimal geodesic from $q$ to $L$.)

(**) If all sectional curvatures of $M$ are $\geq \kappa$, then $q_{L}\left(r^{\prime}\right)=q_{L}(r)$ for some $0<r^{\prime}<r$ only if the normal bundle of $L \hookrightarrow M$ is flat with respect to the induced connection, and $B_{r}(L)$ is (locally) isometric to $\left(L \times D_{r}, g_{\kappa}\right)$. 
In this note, we show that $(*)$ no longer holds in general if one only assumes $\operatorname{Ric}_{M} \geq(m-1) \kappa$ (see also [1] for a related result): In fact, the quotient $q_{L}(r)$ may go to infinity as $r \rightarrow \infty$. Moreover, even if the radial sectional curvatures are $\geq \kappa$-so that $(*)$ must hold- $(* *)$ is no longer true if one replaces $K_{M} \geq \kappa$ by $\mathrm{Ric}_{M} \geq(m-1) \kappa$. More precisely, we have:

\subsection{Theorem. Let $L=\mathrm{C} P^{1}$, and $M=\mathbf{C} P^{2}$. Then}

(a) The normal bundle $E$ of $L \hookrightarrow M$ admits a complete metric of nonnegative Ricci curvature such that

$$
q_{L}(r) \stackrel{\text { def }}{=} \frac{\operatorname{vol} B_{r}(L)}{\operatorname{vol}\left(L \times D_{r}, g_{0}\right)}
$$

goes monotonically to infinity as $r \rightarrow \infty$.

(b) There is a complete metric on $M$ with the following properties:

(1) $L$ is totally geodesically imbedded in $M$.

(2) $\operatorname{Ric}_{M} \geq 3$, and the radial sectional curvatures are $\geq 1$.

(3) $q_{L}(r) \stackrel{\text { def }}{=} \frac{\operatorname{vol} B_{r}(L)}{\operatorname{vol}\left(L \times D_{r}, g_{1}\right)} \equiv 1$ for $r \leq \varepsilon$, provided $\varepsilon$ is suffciently small.

2. Ricci curvature for connection metrics. Let $L=\mathbf{C} P^{1} \hookrightarrow \mathbf{C} P^{2}$ with the standard metric of curvature $1 \leq K \leq 4$. As in [5], we identify a distance tube $B_{r}(L)$ around $L$ with $[0, r] \times S^{3} / \sim$, where all the Hopf fibers are collapsed to a point at $\{0\} \times S^{3}$. Consider the class $d \sigma_{r}^{2}$ of metrics on $S^{3}$ obtained by multiplying the standard metric by $f^{2}(r)$ in the Hopf fiber direction, and by $h^{2}(r)$ on its orthogonal complement. If $f$ is an odd smooth function with $f^{\prime}(0)=1$, and $h$ is even and positive, then the metric $d r^{2}+d \sigma_{r}^{2}$ on $(0, r] \times S^{3}$ extends to $B_{r}(L)$. The standard metric corresponds to $f(r)=(1 / 2) \sin 2 r$ and $h(r)=\cos r$. Using the same vector fields $X_{i}, 0 \leq i \leq 3$, as in [5] (where $X_{0}$ is radial, $X_{1}$ is tangent to the Hopf fiber, and $X_{2}, X_{3}$ are orthogonal to it), we obtain for $R_{i j}:=\operatorname{Ric}\left(X_{i} /\left|X_{i}\right|, X_{j} /\left|X_{j}\right|\right)$ :

$$
\begin{aligned}
& R_{00}=-\frac{f^{\prime \prime}}{f}-2 \frac{h^{\prime \prime}}{h}, \\
& R_{11}=-\frac{f^{\prime \prime}}{f}-2 \frac{f^{\prime} h^{\prime}}{f h}+2 \frac{f^{2}}{h^{4}}, \\
& R_{22}=R_{33}=-\frac{h^{\prime \prime}}{h}-\frac{f^{\prime} h^{\prime}}{f h}+\frac{4 h^{2}-2 f^{2}-h^{\prime 2} h^{2}}{h^{4}}, \\
& R_{i j}=0, \quad i \neq j .
\end{aligned}
$$


The proof is straightforward and will be omitted.

This class of metrics is actually a special case of the following construction: Let $\left(L^{l}, \check{g}\right)$ be a Riemannian manifold, and $\mathbf{R}^{k} \rightarrow E \stackrel{\pi}{\rightarrow} L$ a vector bundle with inner product $\langle$,$\rangle and Riemannian connec-$ tion $\nabla$. Fix $0<r_{0} \leq \infty$, and consider the disk bundle $E^{r_{0}}=$ $\left\{u \in E \mid\langle u, u\rangle<r_{0}\right\}$. If $\mathscr{V}$ denotes the vertical distribution defined by $\pi$, and $\mathscr{H}$ the horizontal distribution determined by the connection, define

$$
g(x, x)=h^{2}(|u|) \check{g}\left(\pi_{*} x, \pi_{*} x\right) \quad\left(x \in \mathscr{H} \cap T_{u} E\right),
$$

where $h$ is an even, smooth, positive function on $\left(-r_{0}, r_{0}\right)$. The fibers of $E^{r_{0}}$ are endowed with a metric given in polar coordinates by

$$
d r^{2}+f^{2}(r) d \sigma^{2},
$$

where $d \sigma^{2}$ is the standard metric on the sphere, and $f$ is an odd, smooth function with $f^{\prime}(0)=1$. We then obtain a metric $g$ on $E^{r_{0}}$ by declaring $\mathscr{H}$ and $\mathscr{V}$ to be mutually orthogonal. The fibers of the bundle are totally geodesic submanifolds in this metric, and the projection $\pi$ restricted to a sphere bundle of radius $r$ becomes a Riemannian submersion with base $\left(L, h^{2}(r) \check{g}\right)$. One can easily compute the Ricci curvatures by using O'Neill's formula for Riemannian submersions and the Gauss equations (cf. also [2]): If $\partial_{r}$ denotes the unit radial vector field (dual to $d r$ ), $v$ a unit vertical vector orthogonal to $\partial_{r}$, and $x$ a unit horizontal vector, then

$$
\begin{gathered}
\operatorname{Ric}\left(\partial_{r}, \partial_{r}\right)=-l \frac{h^{\prime \prime}}{h}-(k-1) \frac{f^{\prime \prime}}{f}, \\
\operatorname{Ric}\left(\partial_{r}, x\right)=\operatorname{Ric}\left(\partial_{r}, v\right)=0, \\
\operatorname{Ric}(v, v)=-\frac{f^{\prime \prime}}{f}+(k-2) \frac{1-f^{\prime 2}}{f^{2}}-l \frac{f^{\prime} h^{\prime}}{f h} \\
+\sum_{i=1}^{l}\left\langle A_{x_{i}} v, A_{x_{i}} v\right\rangle, \\
\operatorname{Ric}(x, x)=-\frac{h^{\prime \prime}}{h}-(l-1) \frac{h^{\prime 2}}{h^{2}}-(k-1) \frac{h^{\prime} f^{\prime}}{h f} \\
+\operatorname{Ric}^{\vee}\left(\pi_{*} x, \pi_{*} x\right)-2 \sum_{i=1}^{l}\left\langle A_{x} x_{i}, A_{x} x_{i}\right\rangle, \\
\operatorname{Ric}(v, x)=\langle(\check{\delta} A) x, v\rangle .
\end{gathered}
$$


Here, $\left\{x_{i}\right\}$ is an orthonormal basis of $\mathscr{H}, A$ is the O'Neill tensor of the submersion with divergence $\check{\delta} A=\sum_{i=1}^{l} D_{x_{i}} A\left(x_{i}, \cdot\right)$ ( $D$ is the Levi-Civita connection of $\left.\left(E^{r_{0}}, g\right)\right)$, and Ric ${ }^{\vee}$ is the Ricci tensor of $\left(L, h^{2}(r) \check{g}\right)$.

Moreover, if $\nabla$ is a Yang-Mills connection, then (cf. [2], p. 243):

$$
\operatorname{Ric}(v, x)=0 \text {. }
$$

In the special case when $E$ is the normal bundle of $\mathbf{C P} \hookrightarrow \hookrightarrow \mathbf{C P}$, let $\nabla$ denote the connection on $E$ induced by the Levi-Civita connection of the symmetric space $\mathbf{C} P^{2}$. Then $\nabla$ is Yang-Mills since the curvature tensor $R^{\nabla}$ is parallel. In particular, (2-9') holds, and it is straightforward to check that (2-5)-(2-9) reduce to $(2-1)-(2-4)$. Notice that the $A$-tensor can be expressed in terms of $R^{\nabla}$, cf. [6].

\section{Proof.}

Proof of 1.1(a). The volume of a distance tube $B_{r}(L)$ with respect to the class of metrics described in $\S 2$ is given by:

$$
\begin{aligned}
\operatorname{vol} B_{r}(L) & =\int_{0}^{r} \operatorname{vol} S_{t}(L) d t \\
& =C \cdot \operatorname{vol}(L) \cdot h^{-l}(0) \cdot \int_{0}^{r} h^{l}(t) f^{k-1}(t) d t,
\end{aligned}
$$

where $S_{t}(L)$ is a distance sphere around $L, \operatorname{vol}(L):=\operatorname{vol}\left(L, h^{2}(0) \check{g}\right)$, and $C$ is the volume of the standard sphere $S^{k-1} \subset \mathbf{R}^{k}$. It thus suffices to find functions $f$ and $h$ such that (2-1)-(2-3) yield Ric $\geq 0$, and $h^{l}(r) f^{k-1}(r) / r^{k-1}=h^{2}(r) f(r) / r \rightarrow \infty$ as $r \rightarrow \infty$. Let $f(r):=$ $r /\left(1+r^{2}\right)^{1 / 2}$, and $h(r):=(r / f(r))^{\alpha}$, where $\alpha$ is any constant in the interval $[1 / 2,1]$. Notice that $q_{L}(r) \rightarrow \infty$ as $r \rightarrow \infty$ if $\alpha>1 / 2$, and $q_{L}(r) \equiv 1$ for $\alpha=1 / 2$.

A straightforward calculation shows that (2-1)-(2-3) become:

$$
\begin{aligned}
R_{0,0} & =\frac{-3(2 \alpha-1)}{\left(1+r^{2}\right)^{2}}+\frac{2 \alpha}{1+r^{2}}\left(2-(\alpha+1) \frac{r^{2}}{1+r^{2}}\right) \\
& =\frac{\alpha}{1+r^{2}}\left(4-\varphi_{\alpha}(r)\right),
\end{aligned}
$$

where $\varphi_{\alpha}(r)=\left(3(2 \alpha-1)+2 \alpha(\alpha+1) r^{2}\right) / \alpha\left(1+r^{2}\right)$. Since $\varphi_{\alpha}$ is an increasing function on $[0, \infty)$ with $\lim _{r \rightarrow \infty} \varphi_{\alpha}(r)=2(\alpha+1) \leq 4$, we conclude that $R_{0,0} \geq 0$.

$$
R_{1,1}=\frac{3-2 \alpha}{\left(1+r^{2}\right)^{2}}+2 \frac{f^{2}}{h^{4}} \geq 0 .
$$




$$
\begin{aligned}
(3-3) R_{2,2}=R_{3,3}= & \frac{-3 \alpha}{\left(1+r^{2}\right)^{2}}+\frac{\alpha}{1+r^{2}}\left(1-\alpha \frac{r^{2}}{1+r^{2}}\right) \\
& +4\left(\frac{f(r)}{r}\right)^{2 \alpha}-2 r^{2}\left(\frac{f(r)}{r}\right)^{2+4 \alpha}-\frac{\alpha^{2} r^{2}}{\left(1+r^{2}\right)^{2}} \\
\geq & \left(1+r^{2}\right)^{-\alpha}\left(4-\left(\psi_{\alpha}(r)+\theta_{\alpha}(r)\right)\right),
\end{aligned}
$$

where $\psi_{\alpha}(r):=2 r^{2} /\left(1+r^{2}\right)^{1+\alpha}$, and $\theta_{\alpha}(r):=\left(3 \alpha+\alpha^{2} r^{2}\right) /\left(1+r^{2}\right)^{2-\alpha}$. One easily checks that the maximum of $\psi_{\alpha}$ equals

$$
\eta(\alpha)=2 / \alpha(1+1 / \alpha)^{1+\alpha} \leq \eta(1 / 2)=4 / 3 \sqrt{3},
$$

for $\alpha \geq 1 / 2$. Moreover, $\theta_{\alpha}$ is a decreasing function if $\alpha \leq 1$, with $\theta_{\alpha}(0)=3 \alpha$. Thus:

$$
R_{2,2}=R_{3,3} \geq\left(1+r^{2}\right)^{-\alpha}(4-(3+4 / 3 \sqrt{3}))>0,
$$

thereby completing the proof of $1.1(\mathrm{a})$.

Proof of 1.1(b). When $h \equiv \cos ,(2-1)-(2-3)$ become:

$$
\begin{aligned}
& R_{0,0}=2-\frac{f^{\prime \prime}}{f} \\
& R_{1,1}=-\frac{f^{\prime \prime}}{f}+2 \frac{f^{\prime} \sin }{f \cos }+2 \frac{f^{2}}{\cos ^{4}} \\
& R_{2,2}=R_{3,3}=1+\frac{f^{\prime} \sin }{f \cos }+\frac{4 \cos ^{2}-2 f^{2}-\sin ^{2} \cos ^{2}}{\cos ^{4}} .
\end{aligned}
$$

We will choose $f$ so that $f(r)=\sin r$ for $r \leq \varepsilon, f(r)=\sin r \cos r$ for $r \geq \pi / 4$, and $R_{i, i} \geq 3$. Define $k:=f / \sin$. (i) and (ii) transform into:

$$
\begin{aligned}
& R_{0,0}=3-\frac{k^{\prime \prime}}{k}-2 \frac{k^{\prime} \cos }{k \sin }, \\
& R_{1,1}=3-\frac{k^{\prime \prime}}{k}-2 \frac{k^{\prime}}{k}\left(\frac{\cos }{\sin }-\frac{\sin }{\cos }\right)+2 k^{2} \frac{\sin ^{2}}{\cos ^{4}} .
\end{aligned}
$$

If $\varepsilon>0$ is sufficiently small, there exists a function $k$ such that $k \equiv 1$ on $[0, \varepsilon], k \equiv \cos$ on $[\pi / 4, \pi / 2]$, and $k^{\prime \prime} \leq 0$. Then $R_{0,0}, R_{1,1} \geq$ 3. To show that $R_{2,2} \geq 3$, observe that, since $f \leq \sin$,

$$
\begin{aligned}
F & \stackrel{\text { def }}{=}\left(4 \cos ^{2}-2 f^{2}-\sin ^{2} \cos ^{2}\right) / \cos ^{4} \\
& \geq\left(4 \cos ^{2}-2 \sin ^{2}-\sin ^{2} \cos ^{2}\right) / \cos ^{4} \stackrel{\text { def }}{=} G .
\end{aligned}
$$


Now, the minimum value of $G=\left(5 / \cos ^{2}\right)-\left(2 / \cos ^{4}\right)+1$ on the interval $[0, \pi / 4]$ is $G(\pi / 4)=3$. Since $R_{2,2}-F=2+\left(k^{\prime} \sin \right) /(k \cos ) \geq 1$, the result follows.

We now proceed to show that the radial sectional curvatures are $\geq 1$ : Let $x \in T_{p} L$, and consider a unit-speed geodesic $\gamma$ originating at $p$ and orthogonal to $L$. If $E$ denotes the parallel field along $\gamma$ with $E(0)=x$, then $J:=h E$ is a Jacobi field along $\gamma$, cf. [3]. Therefore, $R(E, \dot{\gamma}) \dot{\gamma}=-\left(h^{\prime \prime} / h\right) E$, so that $\langle R(E, \dot{\gamma}) \dot{\gamma}, E\rangle \equiv 1$. On the other hand, if $v$ is orthogonal to both $\dot{\gamma}(0)$ and $T_{p} L$, and if $F$ denotes the parallel field along $\gamma$ with $F(0)=v$, then $R(F, \dot{\gamma}) \dot{\gamma}=-\left(f^{\prime \prime} / f\right) F$, and

$$
\langle R(F, \dot{\gamma}) \dot{\gamma}, F\rangle=-f^{\prime \prime} / f=1-\left(k^{\prime \prime} / k\right)-2\left(k^{\prime} / k\right)(\cos / \sin ) .
$$

This last expression is $\geq 1$ and identically 1 on $[0, \varepsilon]$. The same is therefore true for all radial curvatures.

Finally, observe that the comparison space in [4] or [3] has the same volume growth as $\left(L \times D_{r}, g_{\kappa}\right)$. It follows that $q_{L}(r) \equiv 1$ for our choices of $f$ and $h$ when $r \leq \varepsilon$.

\section{Remarks.}

4.1. In 1.1(a), the maximal growth rate for the volume of $B_{r}(L)$ obtained by our method is of order $r^{3}$.

4.2. The maximal distance from $L$ with respect to the metric $g$ from $1.1(b)$ is $\pi /(2 \sqrt{\kappa})=\pi / 2$, where $\kappa$ is the infimum of the radial sectional curvatures and the Ricci curvature. Nevertheless, $(M, g)$ is not symmetric, cf. the remark on p. 322 in [3].

4.3. As the general formulas of $\S 2$ show, one can produce similar examples on other vector bundles. It is, however, essential to have some information about the divergence of the $A$-tensor, cf. (2-9), (2-9').

\section{REFERENCES}

[1] M. Anderson, Short geodesics and gravitational instantons, J. Differential Geom., 31 (1990), 265-275.

[2] A. Besse, Einstein manifolds, Springer Verlag, 1987.

[3] J.-H. Eschenburg, Comparison theorems and hypersurfaces, Manuscripta Math., 59 (1987), 295-323.

[4] E. Heintze and H. Karcher, A general comparison theorem with applications to volume estimates for submanifolds, Ann. Scient. Ec. Norm. Sup., (4)11 (1978), 451-470.

[5] J.-P. Sha and D. G. Yang, Examples of manifolds of positive Ricci curvature, J. Differential Geom., 29 (1989), 95-103. 
[6] M. Strake and G. Walschap, $\Sigma$-flat manifolds and Riemannian submersions, Manuscripta Math. (to appear).

Received September 29, 1989. The first author was supported in part by the Heinrich Hertz Foundation, the second author by a grant from the National Science Foundation.

UNIVERSITY OF CALIFoRNiA, Los ANGELES

Los ANGeles, CA 90024-1555

Current address: University of Oklahoma

Norman, OK 73019 



\section{PACIFIC JOURNAL OF MATHEMATICS EDITORS}

\author{
V. S. VARADARAJAN \\ (Managing Editor) \\ University of California \\ Los Angeles, CA 90024-1555-05 \\ Herbert Clemens \\ University of Utah \\ Salt Lake City, UT 84112 \\ THOMAS ENRIGHT \\ University of California, San Diego \\ La Jolla, CA 92093
}

R. FINN

Stanford University

Stanford, CA 94305

HeRmanN FlaschKa

University of Arizona

Tucson, AZ 85721

VAUGHAN F. R. JONES

University of California

Berkeley, CA 94720

STEVEN KERCKHOFF

Stanford University

Stanford, CA 94305
C. C. MOORE

University of California

Berkeley, CA 94720

Martin ScharlemanN

University of California

Santa Barbara, CA 93106

\section{HAROLD STARK}

University of California, San Diego La Jolla, CA 92093

\section{ASSOCIATE EDITORS}
R. ARENS
E. F. BECKENBACH
B. H. NeumanN
F. WolF
K. YoshidA
(1906-1982)

(1904-1989)
TIONS

UNIVERSITY OF ARIZONA

UNIVERSITY OF BRITISH COLUMBIA

CALIFORNIA INSTITUTE OF TECHNOLOGY

UNIVERSITY OF CALIFORNIA

MONTANA STATE UNIVERSITY

UNIVERSITY OF NEVADA, RENO

NEW MEXICO STATE UNIVERSITY

OREGON STATE UNIVERSITY
UNIVERSITY OF OREGON

UNIVERSITY OF SOUTHERN CALIFORNIA

STANFORD UNIVERSITY

UNIVERSITY OF HAWAII

UNIVERSITY OF TOKYO

UNIVERSITY OF UTAH

WASHINGTON STATE UNIVERSITY

UNIVERSITY OF WASHINGTON 


\section{Pacific Journal of Mathematics}

\section{Vol. 148, No. $1 \quad$ March, 1991}

David Marion Arnold and Charles Irvin Vinsonhaler, Duality and

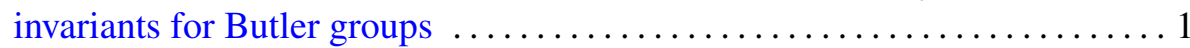

Philippe Delanoë, Obstruction to prescribed positive Ricci curvature . . . . . 11

María J. Druetta, Nonpositively curved homogeneous spaces of dimension

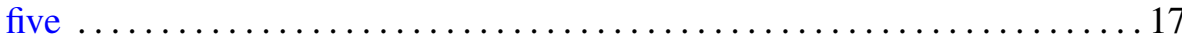

Robert Fitzgerald, Combinatorial techniques and abstract Witt rings III .... 39

Maria Girardi, Dentability, trees, and Dunford-Pettis operators on $L_{1} \ldots \ldots 59$

Krzysztof Jarosz, Ultraproducts and small bound perturbations $\ldots \ldots \ldots \ldots 81$

Russell David Lyons, The local structure of some measure-algebra homomorphisms .................................. 89

Fiona Anne Murnaghan, Asymptotic behaviour of supercuspidal characters of $p$-adic $\mathrm{GL}_{3}$ and $\mathrm{GL}_{4}$ : the generic unramified case $\ldots \ldots \ldots \ldots \ldots 107$

H. Rouhani, Quasi-rotation $C^{*}$-algebras $\ldots \ldots \ldots \ldots \ldots \ldots \ldots \ldots \ldots \ldots \ldots \ldots \ldots \ldots$

Ignacio Sols Lucía, Michał Szurek and Jaroslaw Wisniewski, Rank-2

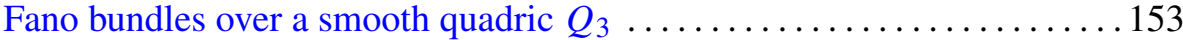

Martin Strake and Gerard Walschap, Ricci curvature and volume

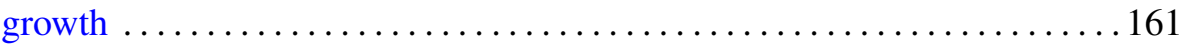

Anton Ströh and Johan Swart, A Riesz theory in von Neumann algebras . . 169

Ming Wang, The classification of flat compact complete space-forms with metric of signature $(2,2)$ 